\title{
O impacto do portal de periódicos da CAPES na produção científica da área de Plasma no Brasil*
}

\section{João de Melo Maricato}

\section{Mestrando do Programa de Ciência da Informação da ECA/USP/Bibliotecário na UNESP}

Analisa uma parcela do sistema de C\&T na área de plasma no Brasil através de indicadores e técnicas cienciométricas. Procura entender a dinâmica da produção científica da área de plasma, dando atenção especial à evolução da produtividade e qualidade das pesquisas entre os anos de 1995 e 2005 (antes e após a implementação do Portal de Periódicos da CAPES). Desse modo, procura visualizar o impacto do Portal de Periódicos da CAPES na produção científica da área de Plasma no Brasil.

Palavras-Chave: Portal de periódicos da CAPES; Avaliação de produção científica; Plasma; Cienciometria; indicadores de C\&T.

\section{The Impact of CAPES Portal of Journals in the Scientific Production of Plasma Science in Brazil}

It evaluates one part of the S\&T system concerning the science of plasma in Brazil through indicators and scientometrics tools. It is a go to understand the dynamic of scientific production of plasma science, approaching in special the productivity evolution and quality of researches within 1995 and 2005 (before and after the implementation of CAPES Portal of Journals). So, it is an overview of CAPES Portal of Journals in the Scientific Production of Brasil Plasma Science.

Baseado no trabalho intitulado "A área de Plasma no Brasil e o impacto do portal de periódicos da CAPES em seu desenvolvimento: um estudo cienciométrico a partir dos grupos de pesquisa cadastrados no CNPq" apresentado à CAPES/MEC/Prêmio para trabalhos sobre o Portal de Periódicos da CAPES em 2006, sendo o premiado na categoria bibliotecário. 
Keywords: CAPES portal of Journals; Scientific production evaluation; Plasma; Scientometrics; S\&T indicators.

Recebido em 17.01.2007 Aceito em 24.04.2007

\section{Introdução}

Especialistas e autoridades governamentais têm tido crescente interesse em análises quantitativas da produção científica, para auxiliar no entendimento da dinâmica da ciência e tecnologia (C\&T) e como instrumentos para planejamento de políticas e tomadas de decisões no campo científico e tecnológico (FAPESP, 2001).

Segundo argumenta Velho (1997), foi na década de 1970 que diferentes países intensificaram o interesse em indicadores científicos, tendo o objetivo de planejar, monitorar e avaliar as atividades de C\&T. Os motivos são diversos, podendo derivar do desenvolvimento institucional do aparato governamental da política de C\&T e da teia de relações estabelecidas com outros segmentos sociais, ou ainda ter relação com o contexto sócio-político-econômico mais geral e com a mudança na visão predominante sobre o papel da C\&T no desenvolvimento dos diversos países. Outra explicação, de diferente natureza, reside na evolução teórica e metodológica das disciplinas que constituem os chamados estudos sociais da C\&T.

Após a Segunda Guerra, os países cientificamente avançados deixaram a cargo dos pesquisadores o poder de decisão no segmento de C\&T. Como progressivamente a C\&T passou a ser considerada portadora de potencialidades para o desenvolvimento econômico e social, houve uma reestruturação institucional do aparato governamental dedicado ao setor. Neste sentido, segundo Velho (1997), foi também nos anos de 1970 que o paradigma da política científica sofreu uma mudança significativa, passando de uma racionalidade ofertista, que caracterizou o período anterior, para uma racionalidade de identificação de prioridades. Diante dessa reestruturação foram incorporados gestores e formuladores de políticas públicas em C\&T que passaram a demandar indicadores quantitativos que demonstrassem as tendências do setor e os resultados das políticas implantadas.

Com a finalidade de suprir essa necessidade, surgem áreas, métodos e técnicas dedicadas a mensurar as informações de C\&T. Segundo Spinak (1998), grande parte dos esforços da ciência da ciência se encontra na elaboração de metodologias para a formulação desses indicadores, onde a ciência é considerada como um sistema de produção de informação, ou seja, um sistema de geração e difusão de conhecimentos que possuem insumos e resultados (inputs e outputs). Neste sentido, Spinak (1998, p. 141), afirma que "a medição dessas categorias - insumos e resultados - são a base dos indicadores científicos". 
Como visto, as primeiras técnicas dedicadas a estudar os indicadores científicos surgiram em meados da década de 70 , e já se encontram em pleno desenvolvimento, ou seja, em idade adulta (CALLON, 1995). Um dos métodos para o estudo do sistema de ciência, tecnologia e inovação é oferecido por um campo disciplinar denominado cienciometria. Conforme argumenta Gregolin (2004, cap. 5, p. 5), os indicadores cienciométricos se ocupam:

[...] do desenvolvimento de metodologias para a construção e a análise de indicadores, com base em abordagem interdisciplinar, envolvendo a bibliometria, a economia, a administração, entre outras. A cienciometria, ou ciência das ciências, abarca o estudo das ciências físicas, naturais e sociais, com o objetivo de compreender sua estrutura, evolução e conexões, de modo a estabelecer relações da ciência com o desenvolvimento tecnológico, econômico e social.

Com base em técnicas cienciométricas, e partindo do conceito de Sistema Nacional de Inovação proposto por Nelson (1993), foi analisada uma parcela do sistema de C\&T na área de plasma ${ }^{1}$ no Brasil. Procurou-se entender a dinâmica da área de plasma de forma ampla e, dentre os indicadores e informações levantadas e analisadas, deu-se atenção especial à evolução da produtividade e qualidade das pesquisas realizadas antes e após a implementação do Portal de Periódicos da CAPES ${ }^{2}$.

\section{Objetivos}

A presente pesquisa teve como objetivos principais investigar a dinâmica da produção científica na área de plasma no Brasil e a influência do Portal de Periódicos da CAPES nessa produção. Para atingir os objetivos deste estudo foi realizada a coleta de dados da área de plasma em diversas fontes de informação (Plataforma Lattes, Diretório dos Grupos de Pesquisa no Brasil, Banco de dados Qualis/CAPES, Currículo

O presente estudo abrange a área de Plasmas de maneira interdisciplinar. No entanto, pode ser generalizada com a definição da área de "Física de Plasmas", que segundo Rezende (1994) se ocupa da investigação de movimentos coletivos de partículas carregadas, elétrons ou íons, ou estados de equilíbrio destas partículas, sujeitas à ação de seus próprios campos. Este conjunto de partículas e campos representa um meio fluido chamado Plasma. Em particular, são tratadas questões como confinamento de plasma, equilíbrio e sua estabilidade, aquecimento e propriedades de transporte, propagação de ondas, interação de partículas com onda, instabilidades, turbulência e caos. São encontrados na natureza, como em descargas elétricas (relâmpagos), na ionosfera, no espaço interplanetário e intersideral, na corona solar, nas estrelas, anãs brancas e pulsares. Eles também são produzidos em laboratórios, como em descargas elétricas, em equipamentos de pesquisa em fusão nuclear, em lasers a gás, dispositivos semicondutores e metálicos e em equipamentos industriais a plasmas.

2 CAPES (Coordenação de Aperfeiçoamento de Pessoal de Nível Superior). A implementação do Portal deu-se no ano 2000, e disponibiliza para universidades e instituições de pesquisa um acervo eletrônico com base de dados referenciais e de resumos - que indexam milhões de artigos em todas as áreas do conhecimento - contando atualmente com mais de 9.500 títulos de periódicos nacionais e internacionais que podem ser acessados através do endereço na Internet www.capes.gov.br. 
Lattes), sua respectiva análise e a construção de indicadores científicos que fornecessem subsídios suficientes para cumprir os objetivos. Dentre os indicadores e informações levantadas e analisadas, destaca-se a caracterização geográfica dos grupos de pesquisa da área de plasma no Brasil; grupos de pesquisa por área do conhecimento; a qualificação de recursos humanos e distribuição por gênero; a produtividade dos pesquisadores e grupos de pesquisa no período entre 1995/2005; identificação do núcleo básico de periódicos na área de plasma, em que os pesquisadores brasileiros mais publicaram no período entre 1995/2005; 0 levantamento da disponibilidade dos periódicos do núcleo básico no Portal de Periódicos da CAPES; a qualidade dos periódicos e conseqüentemente dos artigos publicados entre o período de 1995/2005.

Outros objetivos secundários também foram buscados e se tornaram possíveis com este estudo. Dentre eles, pode-se destacar a análise dos recursos e ferramentas disponibilizadas através da Plataforma Lattes, como o Currículo Lattes, o Diretório dos Grupos de Pesquisa no Brasil do Conselho Nacional de Desenvolvimento Científico e Tecnológico (CNPq) e o Banco de dados Qualis da CAPES para a construção de indicadores de C\&T. O uso e análise desses recursos certamente puderam cooperar com a elaboração de metodologias mais eficazes para a formulação de indicadores de C\&T.

Finalmente, as informações resultantes desta pesquisa poderão ser utilizadas para a formulação de políticas públicas para a área de Plasma. Tanto políticas de informação (como é o caso do Portal de Periódicos da CAPES), quanto para políticas de financiamento da área como um todo.

\section{Metodologia e limitações}

Como visto anteriormente, a medição dos insumos e resultados (inputs e outputs) são a base dos indicadores cienciométricos. No entanto, este estudo se limitou a estudar alguns indicadores de resultados (outputs). O mais próximo de indicadores de insumos (inputs) a que se chegou foi de uma parcela de infra-estrutura para pesquisa, ou seja, a assinatura de periódicos pela CAPES. Não foi analisada a infra-estrutura para pesquisa como bibliotecas, laboratórios e equipamentos, financiamento em Pesquisa e Desenvolvimento (P\&D), etc. Em abordagens mais aprofundadas, é importante a análise dos intputs, já que, como demonstra a maioria dos estudos, no plano institucional, há um conjunto de fatores que se correlacionam com a produtividade e qualidade (RUSHTON; MELTZER, 1981 apud MEADOWS, 1999, p. 88-89 é necessário as p.?).

Primeiramente foram extraídas as informações sobre os grupos de pesquisa na área de plasma no Brasil. A busca deu-se entre os dias 15 e 25 de fevereiro de 2006 na base corrente do Diretório dos Grupos de Pesquisa no Brasil da Plataforma Lattes, disponível na página da internet 
através do endereço http://lattes.cnpq.br/index.htm ${ }^{3}$. Nas buscas foram utilizados os termos plasma e plasmas, nas áreas de engenharia e ciências exatas e da terra ${ }^{4}$. Com esse critério de busca, notamos que além dos grupos que atuam na área de plasma, também foi selecionado um grupo de pesquisa que atua na área de Física das partículas elementares, mais precisamente na área de Quark Gluon Plasma. Considera-se que a presença de tal grupo não tenha afetado grandemente os resultados deste estudo, já que se trata de um grupo composto de apenas 6 pesquisadores, também pertencente à área de Física.

Optou-se por não buscar grupos na área de ciências da saúde pelo fato de a busca retornar muitos grupos que atuam, por exemplo, na área de plasma sanguíneo. Mesmo que possa haver algum grupo na área de ciências da saúde que atue com plasma na análise, síntese, tratamento, etc. de materiais, acredita-se que os resultados deste trabalho não seriam significativamente alterados.

A busca de informações estatísticas a partir do Diretório dos Grupos de pesquisa possui algumas limitações. Ferreira (2004) considera o Diretório como "referência obrigatória nos levantamentos de recursos humanos em P\&D". No entanto, o mesmo autor sinaliza que o argumento de que o seu escopo ultrapassa a definição estrita de $P \& D$ não é a limitação mais relevante, e sim outras, como a dificuldade de se conhecer sua efetiva cobertura, 0 fato de suas estatísticas consolidadas incluírem uma parcela variável de dupla contagem (com o nível de detalhe da informação), de suas informações básicas serem prestadas voluntariamente pelos pesquisadores (pode-se supor que nem todos as prestem), etc. No entanto considera que "sua base de dados é extremamente rica e permite uma primeira aproximação da dimensão do pessoal alocado em atividades de P\&D" (FERREIRA, 2004, cap. 4, p. 18).

Outra fonte de informação importante para a realização do presente estudo foi a obtenção do Currículo Lattes do pesquisador na página da internet localizada no endereço http://lattes.cnpq.br/. Após a identificação dos grupos de pesquisa, foram localizadas, dentro de cada grupo, as linhas de pesquisa com alguma relação com a área de plasma e, desse modo, identificados os pesquisadores envolvidos com a área. Com os nomes dos pesquisadores em mãos, procedeu-se a busca do Currículo Lattes do pesquisador (entre os dias 15 e 27 de fevereiro de 2006), a identificação e coleta dos artigos científicos publicados por cada autor. Os artigos científicos utilizados neste estudo foram limitados ao período entre 1995 e 2005 (cinco anos antes e cinco anos após a implementação do Portal de Periódicos da CAPES).

3 Os grupos de pesquisa não-atualizados não aparecem nas buscas realizadas na base de dados dos Grupos de Pesquisa no Brasil do CNPq. Segundo a CAPES, um grupo perde a certificação quando fica mais de 12 (doze) meses sem sofrer atualização. Portanto, caso haja algum grupo de pesquisa na área de plasma nesta condição (sem atualização nos últimos 12 meses) o mesmo não seria contemplado neste estudo.

4 Segundo a CAPES, as buscas são feitas sobre o nome do grupo, título da linha e palavraschave da linha de pesquisa. 
A avaliação da produtividade com base na publicação de artigos científicos certamente não é perfeita, mas pode ser considerada, no momento, como uma das melhores alternativas disponíveis. Alguns problemas desse tipo de avaliação podem influenciar estudos deste tipo. Por exemplo, é bastante provável que dentre os artigos publicados pelos pesquisadores que atuam em grupos de plasma, existam artigos que não tenham relação alguma com plasma.

Para chegar a um índice de grupos de pesquisa mais produtivos, foi necessária a exclusão dos artigos que apareciam mais de uma vez no mesmo grupo (os principais motivos dessa repetição são os artigos publicados em parceria com demais membros de um mesmo grupo).

$\mathrm{Na}$ construção de um índice de periódicos onde os pesquisadores da área de plasma mais publicam, foram excluídos todos os artigos repetidos, sendo contados uma única vez, independentemente de estarem presentes em um ou mais grupos de pesquisa. Com esse indicador, identificou-se uma espécie de núcleos de periódicos da área, com alguma semelhança à famosa Lei de Bradford (BRADFORD, 1934). Essa Lei possibilita, em certa medida, o desenvolvimento de políticas de aquisição e descarte de periódicos (GUEDES; BORSCHIVER, 2005).

A construção de indicadores baseados na publicação dos periódicos foi uma atividade de extrema complexidade e dificuldade devido a problemas originados, sobretudo, no preenchimento do Currículo Lattes. Considerando essas dificuldades, poderá haver uma pequena margem de erro nos dados apresentados (principalmente quanto aos indicadores em que o título do periódico era essencial). No entanto, nada que comprometa a sua validade.

A determinação da qualidade dos artigos científicos publicados pelos pesquisadores na área de plasma teve como base a qualidade dos periódicos utilizados na veiculação desses artigos. Utilizamos a base de dados Qualis: Classificação de Periódicos, Anais, Jornais e Revistas, versão 1.0 de 2004 (CAPES, 2004) $^{5}$. Essa base foi concebida pela CAPES objetivando a avaliação dos programas de pós-graduação, no entanto, pôde ser perfeitamente adotada e adaptada à presente pesquisa. Sobre o Qualis, a CAPES (2004) esclarece que os veículos de divulgação são enquadrados em categorias indicativas da qualidade ( $A, B$ ou $C$ ) e do âmbito de circulação dos mesmos (local, nacional ou internacional). A combinação dessas categorias compõe indicativos da importância do veículo utilizado e, por inferência, do próprio trabalho divulgado.

Como no Qualis, um mesmo periódico pode ser classificado em duas ou mais áreas distintas e receber diferentes avaliações. Para efeitos dessa análise, foram necessários alguns critérios especiais, já que foram

5 O Qualis é uma classificação feita pela CAPES dos veículos utilizados pelos programas de pósgraduação para a divulgação da produção intelectual de seus docentes e alunos, cujo objetivo é atender às necessidades específicas da avaliação da pós-graduação realizada pela agência. Revista e atualizada anualmente, essa classificação é feita por 44 comissões de consultores, cada qual focalizando um conjunto específico de áreas do conhecimento, e se baseia nas informações fornecidas pelos programas, por meio do Coleta de Dados/CAPES, sobre os trabalhos publicados por seus docentes e discentes. 
identificados na área de plasma no Brasil grupos de pesquisa da área de Física, Engenharia de Materiais e Metalurgia, Engenharia Mecânica, Engenharia Elétrica, Química, Geociências e Engenharia Aeroespacial. Com base na área do grupo de pesquisa, os artigos foram classificados dentro de suas respectivas áreas (quando da ocorrência de um mesmo periódico ter sido classificado em mais de uma área). Por exemplo, no caso de periódicos classificados na área de Astronomia/Física e na área de Engenharia de Materiais e Metalurgia (com classificações diferentes), foi necessária a identificação da área de atuação do grupo responsável pelo artigo para determinar a classificação correspondente. Os artigos que não se enquadravam em nenhuma das áreas, que não foram localizados ou que não estão classificados no banco de dados Qualis, receberam a identificação genérica $N C$ (não classificado).

No caso da classificação Qualis das Engenharias (que está dividida em I, II, III e IV), deu-se preferência às classificações relacionadas às Engenharias II por abranger as engenharias de materiais e metalurgia (a mais representativa quando considerado o número de grupos de pesquisa na área de engenharia). Quando o periódico não foi classificado na área de Engenharias II, foi considerada a área de Engenharias III, que agrupa as áreas de Engenharia Aeroespacial e Mecânica.

\section{Resultados}

\section{Distribuição geográfica dos grupos de pesquisa}

Com base na coleta de dados do Diretório dos Grupos de Pesquisa do CNPq, pode-se visualizar com maior clareza como está estruturada a área de plasma no Brasil. Foram localizados 40 grupos de pesquisas que em maior ou menor grau atuam com tecnologias relacionadas a plasma. Os grupos estão distribuídos por relativamente poucas regiões e estados brasileiros (9 Estados e 4 Regiões). A grande maioria dos grupos de pesquisa está distribuída por universidades do Estado de São Paulo $(45 \%)$. Seguido por uma quantidade consideravelmente alta de grupos, está o Estado do Paraná com $18 \%$ dos grupos de pesquisa. Rio Grande do Sul e Santa Catarina contam, cada um, com $10 \%$ dos grupos. Os demais Estados variam entre $3 \%$ e $5 \%$ dos grupos de pesquisa na área de plasma no Brasil.

\section{Grupos de pesquisa por tipo de financiamento}

As instituições de pesquisa brasileiras que atuam na área de plasma estão distribuídas entre públicas (estaduais e federais) e privadas. Quatorze destas instituições são mantidas pelos governos estaduais. São 21 as instituições mantidas com recursos federais que desenvolvem atividades de pesquisa na área de plasma no Brasil.

Seguindo uma tendência nacional, nota-se que os investimentos privados na área de P\&D são bastante baixos também na área de plasma. São apenas 5 grupos de pesquisa vinculados a instituições privadas. 


\section{Distribuição de grupos de pesquisa por área do conhecimento}

Nos levantamentos, limitou-se em buscar dados das "grandes áreas" de "ciências exatas e da terra" e "engenharias", como citado anteriormente. Dos quarenta grupos com alguma linha de pesquisa direcionada à área de plasma, 22 são da grande área de "ciências exatas e da terra" e os demais (18) são da grande "área de engenharia".

Os grupos da grande área de pesquisa "ciência exatas e da terra" estão distribuídos por três "áreas de pesquisa": física, química e geociências. A esmagadora maioria dos grupos está concentrada na área de física, com 19 grupos, enquanto 2 grupos são da área de química e 1 da área de geociências.

\section{Grupos, linhas de pesquisa e pesquisadores envolvidos na área de plasma}

Os 40 grupos identificados estão subdivididos em 88 linhas de pesquisas (média de 2,2 linhas de pesquisa por grupo). A quantidade de linhas de pesquisa varia bastante dentre os grupos de pesquisa. Enquanto existem grupos com apenas uma linha de pesquisa dedicada a área de plasma, diversos fazem parte de grupos de pesquisa mais genéricos, ou seja, que englobam uma diversidade de outras áreas de pesquisa. Também foram identificados grupos (com até 8 linhas de pesquisa) muito mais específicos e com 0 foco totalmente voltado para pesquisas envolvendo plasma.

O número total de pesquisadores com alguma atividade relacionada à área de plasma nos grupos de pesquisa cadastrados no CNPQ é de 154, que corresponde a uma média de aproximadamente 3,8 pesquisadores por grupo de pesquisa (na TAB. 1 são descritos como 163 o número total de pesquisadores pelo fato de 9 pesquisadores participarem de 2 grupos de pesquisa e por não considerar coerente excluí-los aleatoriamente de um dos grupos de pesquisa).

A alta qualificação dos pesquisadores na área de plasma é invejável. Apenas três pesquisadores (2\%) não possuem doutorado completo. Dentre os que possuem doutorado completo, vinte (14\%) são Livredocentes e cinqüenta e dois (36\%) realizaram Pós-Doutorado.

A distribuição de pesquisadores por gênero não foge muito da área de C\&T em geral, predominando cientistas do sexo masculino nessa área do conhecimento ( $83 \%$ versus $17 \%$ ).

O número de pesquisadores por grupo de pesquisa é bastante heterogêneo, ou seja, enquanto alguns grupos possuem apenas um pesquisador na área, como é o caso do grupo de pesquisa do CEFEET/RN, outros contam com uma equipe de pesquisadores muito maior, como é o caso do grupo de pesquisa vinculado ao ITA com o total de 15. 


\section{A produtividade dos grupos de pesquisa no período entre 1995 e 2005}

Para se chegar a um índice de grupos de pesquisa que mais produziram no período, foi necessária a exclusão dos artigos que apareciam mais de uma vez no mesmo grupo (o principal motivo dessa repetição é o fato de existirem artigos publicados em parceria com demais membros do grupo). O número total de artigos publicados no período entre 1995/2005, seguindo-se este critério, é de 2055. Cabe lembrar que os artigos publicados em colaboração (co-autoria) com pesquisadores de outros grupos não foram excluídos. Por exemplo, caso um pesquisador da UFRN tenha publicado um artigo em conjunto com um pesquisador da UNESP, esse artigo foi contabilizado em ambos os grupos.

Considerando os artigos publicados em co-autoria com demais grupos, independentemente do Estado, chega-se ao total de 2673 artigos publicados no período de 1995/2005. 
TABELA 1 - Grupos de pesquisa por ordem de quantidade de artigos publicados por pesquisador (1995/2005)

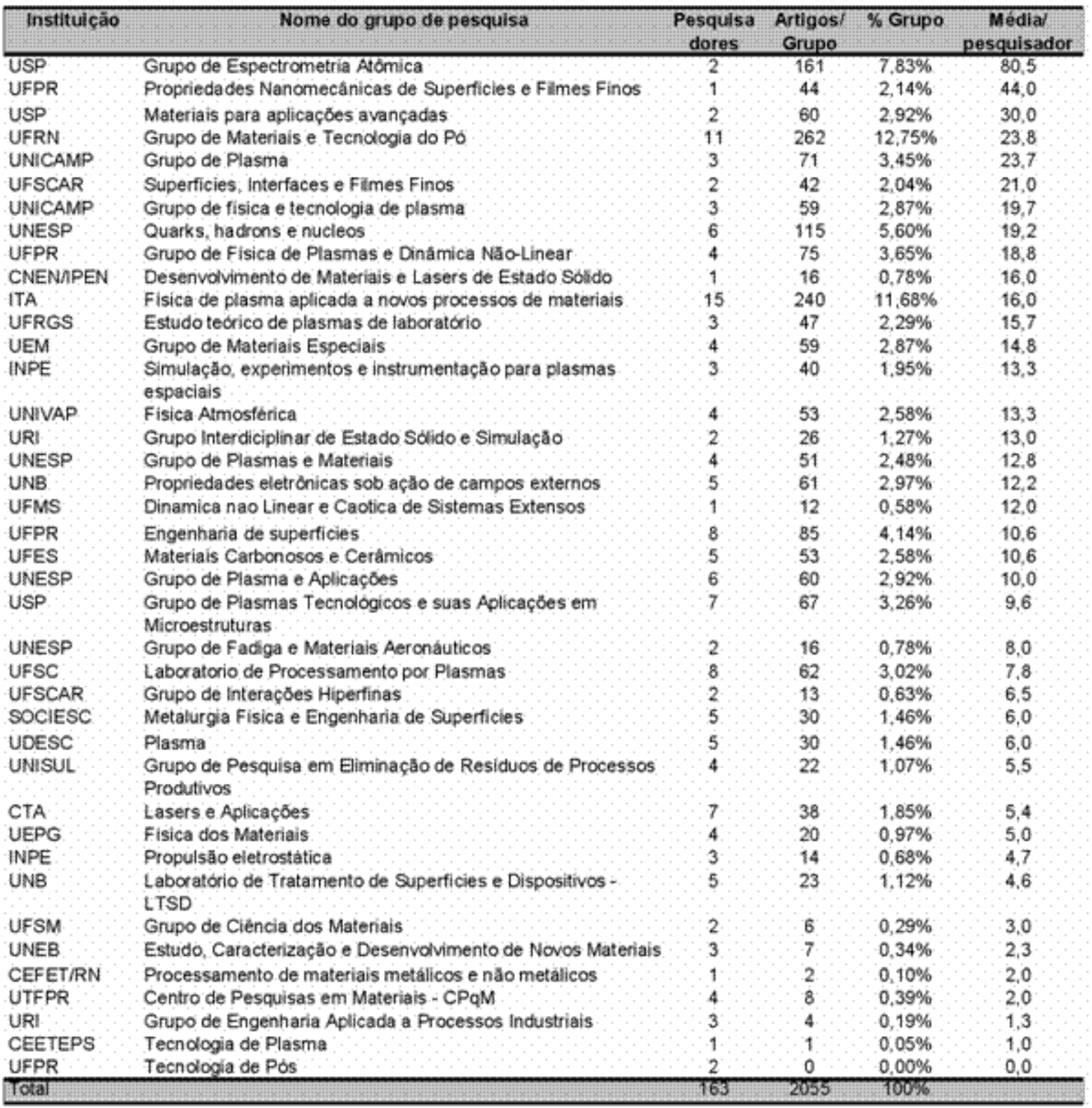

Na TAB. 1 estão classificados, em ordem decrescente, os grupos de pesquisa mais produtivos, ou seja, os grupos que mais publicaram artigos no período de 1995/2005 com relação ao número de pesquisadores. Com base nesse critério, constatou-se que o Grupo de Espectrometria Atômica da USP é o mais produtivo. Com apenas 2 pesquisadores, publicaram no período de 1995/2005 o total de 161 artigos (7,83\% do total), sendo a média por pesquisador no período (1995/2005), de 80,5 artigos publicados.

Em seguida estão os grupos de Propriedades Nanomecânicas de Superfícies e Filmes Finos da UFPR, com média de 44 artigos publicados por pesquisador, e o grupo de Materiais para aplicações avançadas da USP, com média de 30 artigos por pesquisador. 


\section{Evolução da publicação de artigos entre o período de 1995/2005}

Analisando os 2673 artigos publicados ao longo do período de 1995/2005, notamos que há, apesar das oscilações, um crescimento médio constante (GRÁF. 1). Praticamente todos os estados brasileiros tiveram aumento da produtividade, elevando substancialmente o número de artigos publicados no período.

Com exceção de estados aparentemente pouco tradicionais em pesquisa na área de plasma, como Bahia e Mato Grosso do Sul (que tiveram decréscimo na sua produtividade), todos os outros estados tiveram aumento - em alguns casos bastante expressivos - na publicação de artigos científicos quando comparados os períodos de 1995/1999 versus 2001/2005.

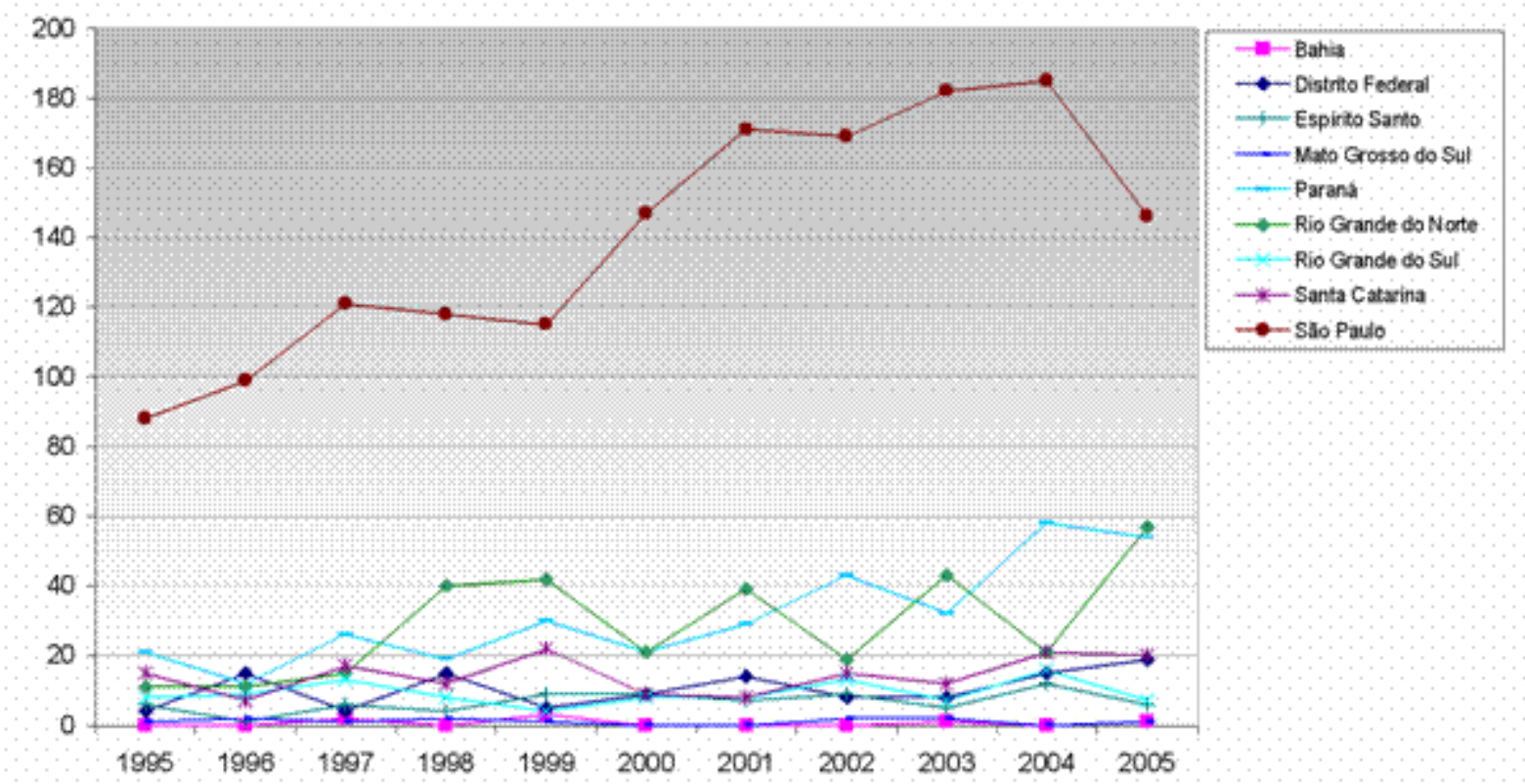

GRÁFICO 1 - Evolução da publicação de artigos (1995/2005)

Com o melhor índice de aumento de produtividade entre o período de 1995/1999 versus 2001/2005 estão os grupos de pesquisa do estado do Paraná. No primeiro período analisado (1995/1999) foram publicados 108 artigos e no segundo período (2001/2005) foram publicados 216 , ou seja, um aumento de $100 \%$ com relação ao período anterior. Os grupos de pesquisa do Distrito Federal publicaram 43 artigos no período entre 1995/1999 e 64 no período entre 2001/2005, correspondendo a um aumento de $49 \%$. Os grupos de pesquisa do Espírito Santo e do Rio Grande do Norte tiveram um aumento de $50 \%$ cada um na produtividade, 
partindo respectivamente de 26 para 39 artigos publicados no Espírito Santo e de 119 para 179 artigos no Rio Grande do Norte.

Os grupos de pesquisa do Estado de São Paulo saltaram de 541 para 853 artigos publicados, o que significa aproximadamente $58 \%$ de aumento no segundo período. No Rio Grande do Sul os artigos publicados saltaram de 42 para 51 (21\% de aumento com relação ao primeiro período). Os Grupos de pesquisa do estado de Santa Catarina publicaram apenas $4 \%$ a mais (passando de 73 para 76 artigos) (GRÁF. 1).

Somando os artigos de todos os grupos no período entre 1995/1999, chegamos a 964 artigos publicados. No período de 2001/2005 foram publicados 1485 artigos. Comparando os dois períodos, nota-se que no último houve um aumento de aproximadamente 54\%. Trata-se de um aumento de produtividade extraordinário a partir do ano 2000, data de implantação do Portal de Periódicos da CAPES.

\section{Periódicos onde os pesquisadores da área de plasma mais publicaram}

Para identificação dos periódicos onde os pesquisadores da área de plasma mais veicularam seus artigos no período de 1995/2005, foi necessário excluir todos os artigos que apareciam mais de uma vez, independentemente do(s) grupo(s) de pesquisa(s) a que pertencia(m). Ou seja, os artigos que apareciam em mais de um grupo de pesquisa foram contados uma única vez. Com base nesse critério, chegou-se a um total "real" de 1964 artigos publicados no período entre 1995 e 2005, com uma média de 12,5 artigos por pesquisador, o que corresponde a aproximadamente 49 artigos por grupo de pesquisa.

Nota-se que os 1964 artigos identificados estão dispersos em aproximadamente 480 periódicos diferentes (média de aproximadamente 4 artigos por periódico). A variação do número de artigos por periódico é de 1 a 73 (aproximadamente $0,05 \%$ à 3,72\% respectivamente). Na Tabela 2, são apresentados 62 periódicos que publicaram entre 8 e 73 artigos de pesquisadores da área de plasma no Brasil. Mesmo que seja alta a dispersão de artigos por periódicos, a respectiva relação pode ser um bom indicativo do núcleo de periódicos da área de plasma, já que agrupam aproximadamente $60 \%$ de todos os artigos publicados no período.

\section{Disponibilidade dos periódicos do núcleo básico no portal de periódicos da CAPES}

Com base nos periódicos em que os pesquisadores da área de plasma mais publicaram seus artigos no período entre 1995/2005 e a respectiva identificação do que aqui se denominou núcleo de periódicos da área de plasma, foi possível a identificação dos periódicos que estão disponibilizados através do Portal de Periódicos da CAPES (independentemente do acesso ser livre ou pago). 
Esse levantamento possibilita visualizar a disponibilidade e acessibilidade tanto do periódico quanto dos artigos publicados pelos pesquisadores. Com a identificação do núcleo básico de periódicos da área de plasma, notou-se que a maioria encontra-se disponibilizada via Portal de Periódicos. Assim, dos 62 periódicos que representam o "núcleo básico", 46 (aproximadamente 74\%) são disponibilizados via Portal. Isso corresponde a aproximadamente $48 \%$ do total de artigos publicados no período. No entanto, uma parcela menor, mas considerável, não se encontra disponibilizada no Portal, ou seja, 16 periódicos (aproximadamente 26\%) que corresponde a $12,7 \%$ do total de artigos publicados no período. Dentre os periódicos não disponíveis, o que mais chama a atenção é o Materials Science Fórum, por ser o número 2 no ranking de periódicos onde os pesquisadores da área mais publicaram (55 artigos, ou seja, 2,8\% do total). Os periódicos do núcleo básico disponibilizados e não disponibilizados via Portal de Periódicos da CAPES são apresentados na TAB. 2. 
TABELA 2 - Periódicos em que os pesquisadores da área de Plasma mais publicaram seus artigos no período entre 1995/2005 e a disponibilidade no Portal de Periódicos da CAPES

\begin{tabular}{|c|c|c|c|}
\hline Titulo & Quantidade & \% & $\begin{array}{l}\text { Oisporiver } \\
\text { no Portal }\end{array}$ \\
\hline Brazilua Joumal of Physiçs & 8.73 & $\overline{3.72 \%}$ & $\sin$ \\
\hline Malenals Solence Forum & 55 & $2,80 \%$ & Nob: \\
\hline Surtace and Coatings Technology. & 51 & $2.60 \%$ & Sim \\
\hline Thin Solid Filin s & 47 & $2,39 \%$ & Sin \\
\hline Physics ot Plasmas. & 35 & $-1,78 \%$ & $\sin$ \\
\hline Acta Microscopica & 34 & $1,73 \%$ & Sin \\
\hline \multicolumn{4}{|l|}{ Physical Review E - Statistical Physics, Plasm as, Fluids and Related. } \\
\hline Interdisclplinary Topics & 34 & $1.73 \%$ & Siro. \\
\hline Journal of Vacuum Science \& Technology A - Vacuum Surlaces and & & & \\
\hline Fins $\$$ : & 31 & $1.58 \%$ & Sim \\
\hline Materials Researeh Revista lbero Americana de Materiais & 31 & $1.58 \%$ & Sim. \\
\hline Materials Science and Engineering $\mathrm{A}$ : & 30 & $1.53 \%$ & $\sin$ \\
\hline Joumal of Appled Physics & 27 & $1,37 \%$ & Sin. \\
\hline Chaos. Solitons and Fractals & 26 & $1.32 \%$ & Sin \\
\hline Spectrochim ica Acta Pant B-Atom ic Spectroscopy. & 25 & 1,2796 & $\sin$ \\
\hline Advances in space Research & 24 & $1.22 \%$ & $\sin$ \\
\hline Joumal of Physics D - Applied Physics & 24 & 1,22 先 & Sin \\
\hline Revista Brasileira de Aplicaçós de Vacuo & 23 & 1,1796 & Sim \\
\hline Physica A - Statistical and Theoretical Physics & 21 & 1,0746 & Năo: \\
\hline Physiosi Review $B$ - Condensed Matter and Materiais Physics & 21 & $1,07 \%$ & Sin' \\
\hline \multicolumn{4}{|l|}{ Talenta. The lnternational Journal of Pure and Applied Analytical } \\
\hline Chemistry & 20. & $1,02 \%$ & $\sin$ \\
\hline joumal of Analytical Atom ic Spectrometry. & 19 & $0,97 \%$ & Nato \\
\hline Metalurgia 8 Moteribis & 19 & $0.97 \%$ & $\sin$ \\
\hline Physics letters B Nuclear Physics and Particie Physics & 19 & $0.97 \%$ & Sim. \\
\hline Diam ond and Related Materials & 18 & $0.92 \%$ & Sim \\
\hline Joumal of geophysical Research & 18 & $0.92 \%$ & Năo \\
\hline Quimica Nova & 18 & $0.92 \%$ & $\sin$ \\
\hline Physical Review C - Nuclear Physics & 17 & $0.87 \%$ & $\sin$ \\
\hline Applied Surface science & 16 & $0.81 \%$ & Sim \\
\hline Journal of Engineering Physics And Therm ophysics & 16 & $0.81 \%$ & Sin \\
\hline Journal of Materials Science & 16 & $0.81 \%$ & $\sin$ \\
\hline Joumal of Engines ring Physics and Thermophysics & 15 & $0.76 \%$ & Sin \\
\hline Joumal of The Brazilian Chem ic sol society & 15 & 0.7696 & Şim \\
\hline Nuclear Instruments and Methods in Physics Research $B$ & 15 & $0,764 \%$ & Sim. \\
\hline Phystoa Status solidi B & 15 & $0.76 \%$ & Nato \\
\hline Reviste Soldagem $s$ inspeçăo & 15 & 0,769 & Näo \\
\hline Annales 6 oppysicae & 14 & 0.7196 & Sim: \\
\hline Physics Letters $\mathrm{A}$ & 14 & $0,71 \%$ & $\sin$ \\
\hline Plasm a Physics And Controllod Fusion & 14 & $0.71 \%$ & Sim \\
\hline IEEE Transactions on Plasma Science - & 13 & $0.66 \%$ & $\sin$ \\
\hline Journal of the Physical Sochety of Japan & 13 & $0.86 \%$ & Nab \\
\hline Coramica & 12 & $0.61 \%$ & Sin \\
\hline Joumal of Magnetism and Magnetic Materials & 12 & $0.61 \%$ & Sim \\
\hline Nuciear Physics A Nuclear and Hadronic Physics. & 12 & $0,61 \%$ & $\sin$ \\
\hline Japanese Journal of Applied Physics & 11 & $0.56 \%$ & Nab \\
\hline Journal of Atm ospheric and Solar -Terrestrial Physics & 11 & $0.56 \%$ & Sin : \\
\hline Journal of Physics 6 Nuclear and Particle Physios " & 11. & $0.56 \%$ & $\sin$ \\
\hline Key Engineering Materials & 11 & $0.56 \%$ & Nato \\
\hline Mieroelectronics Joumal : & 11 & $0.56 \%$ & Sim. \\
\hline Hyperfine interactions & 10 & 0.5196 & Sim \\
\hline Journal of Non-Crystatline Solids & 10 & $0.51 \%$ & $\sin$ \\
\hline Physica C - Superconductivity and its Applications & 10 & $0,51 \%$ & $\sin$ \\
\hline Physica Status solidi A & 10 & $0.51 \%$ & Näó \\
\hline Applied Physics Letters & 9 & $0.46 \%$ & $\sin$ \\
\hline Atmosphenc and Terrestrial Physics : & 9 & $0,46 \%$ & $\sin$ \\
\hline International joumal of Powder Metaliurgy. & 9 & $0.46 \%$ & Näo. \\
\hline Physica D-Nonlinear Phenom ena & 9 & $0,46 \%$ & Sim. \\
\hline Wear & 9 & 0.4646 & $\sin$ \\
\hline Advances in Pow der Metallurgy \& Particulate Materials & 8 & $0.41 \%$ & Ná \\
\hline Analytica Chimica Acto & 8 & $0,41 \%$ & $\sin$ \\
\hline European Physics Journat A - Applied Physics & 8. & $0,41 \%$ & Nä \\
\hline Geophysical Research Lotters & 8 & $0.41 \%$ & Nab. \\
\hline Revista Fisica Aphicada e instrum entaçó & 8 & $0.41 \%$ & NÁ \\
\hline Vacuum & 8 & $0.41 \%$ & Näo. \\
\hline
\end{tabular}




\section{Qualidade dos periódicos em que os pesquisadores veicularam seus artigos}

Utilizando-se a base de dados Qualis: Classificação de Periódicos, Anais, Jornais e Revistas (versão 1.0 de 2004) da CAPES, chegou-se a um indicador de qualidade dos periódicos utilizados na veiculação desses artigos e, por inferência, dos artigos científicos publicados pelos pesquisadores na área de plasma.

Os 1964 artigos publicados no período (1995/2005) foram classificados em A, B, C e NC (não classificado). Dessa forma, foram identificados 1170 , o que corresponde a aproximadamente $60 \%$ de artigos publicados em periódicos $A$ ( $51 \%$ em periódicos estrangeiros e $9 \%$ em periódicos nacionais), 342 (18\%) de artigos publicados em periódicos $B$ (10\% em periódicos estrangeiros e $8 \%$ em periódicos nacionais), 108 (5\%) de artigos publicados em periódicos C (4\% em periódicos estrangeiros e $1 \%$ em periódicos nacionais), $344(18 \%)$ de artigos publicados em periódicos NC (14\% em periódicos estrangeiros e $4 \%$ em periódicos nacionais).

Comparando a qualidade dos periódicos em que os pesquisadores da área de plasma mais publicaram nos períodos de 1995/1999 versus 2001/2005, nota-se um considerável aumento no segundo período, após a implementação do Portal de Periódicos da CAPES. Os artigos publicados em periódicos com classificação A no período de 2001/2005 cresceram aproximadamente 34\% com relação ao período de 1995/1999. Os artigos publicados em periódicos com classificação B cresceram aproximadamente $48 \%$ e os publicados em periódicos com classificação C cresceram aproximadamente $49 \%$. O crescimento dos artigos publicados com a classificação NC permaneceu praticamente estável (4\%) em comparação com o período anterior (GRÁF. 2). 


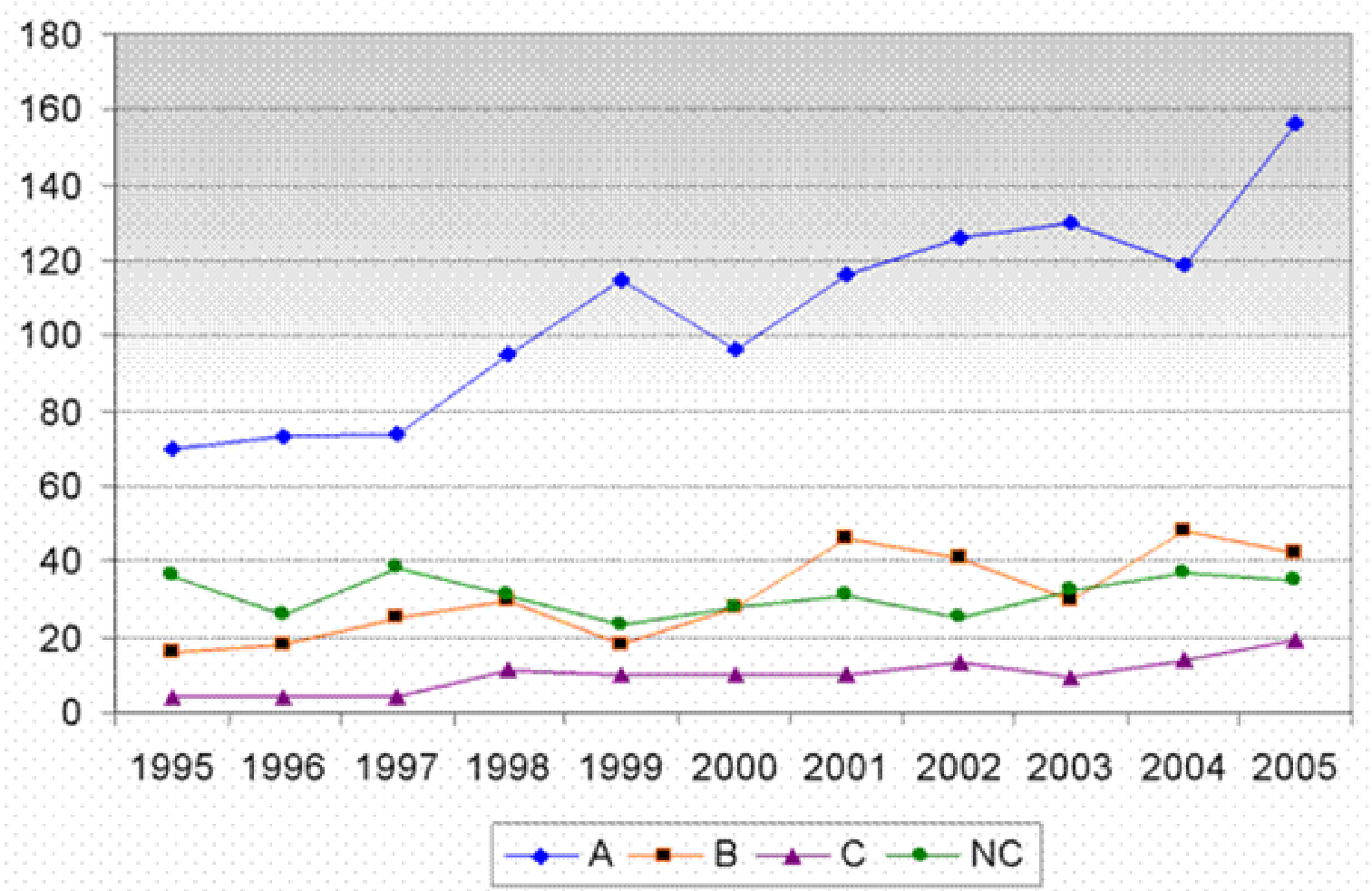

GRÁFICO 2 - Qualidade dos periódicos onde os pesquisadores veicularam seus artigos (1995/2005)

\section{Conclusões e recomendações}

Com a coleta de dados do Diretório dos Grupos de Pesquisa do CNPq foi possível visualizar com maior clareza como está estruturada a área de plasma no Brasil. Apesar das limitações, o Diretório dos Grupos de Pesquisa no Brasil do CNPq pode ser considerado uma boa fonte de informações para a construção de indicadores cienciométricos (sobretudo no caso de recursos humanos). Demonstrou-se extremamente útil para a identificação de pesquisadores e áreas de pesquisa específicas, podendo ser considerado uma ferramenta auxiliar nos estudos de prospecção tecnológica. Uma das limitações encontradas é o fato dos grupos de pesquisa não-atualizados nos últimos 12 meses não aparecem nas buscas. Para estudos semelhantes ao presente, consideramos importante que seja revista essa limitação, mesmo sabendo que o ideal seja a atualização periódica dos grupos de pesquisa por parte dos pesquisadores.

Outra fonte de informações extremamente importante para este estudo foi a Base de Dados denominada Currículo Lattes também presente na Plataforma Lattes do CNPq. Apesar das limitações, o Currículo Lattes está gradativamente se tornando uma base consistente, podendo ser considerada como referência obrigatória pela grande potencialidade em subsidiar análises bibliométricas e cienciométricas de produção científica 
no contexto nacional (e quem sabe, em breve, no contexto latino americano).

Nota-se que a construção de indicadores com base em informações captadas através do Currículo Lattes (principalmente quanto a periódicos) foi uma atividade de extrema complexidade e dificuldade devido a problemas originados, sobretudo, no seu preenchimento. A falta de padronização dos elementos, erros no preenchimento e ausência de elementos essenciais para a identificação da publicação (autor, título do artigo, título do periódico, data de publicação, local de publicação, etc.) foram os principais problemas encontrados. Outra dificuldade detectada foi a existência de alguns trabalhos indicados como artigos científicos, sendo que na verdade tratavam-se de outros tipos de trabalhos, como por exemplo, trabalhos apresentados em congressos e outros eventos, assim como trabalhos publicados em revistas de divulgação/jornalismo científico.

O que se considera mais preocupante é que essas dificuldades poderão vir a inibir a utilização do Currículo Lattes para fins de avaliações da produção científica devido, principalmente, à grande dificuldade de tratamento dos dados. Acredita-se que é preciso buscar soluções para estas limitações do Currículo Lates, caso tenhamos interesse em utilizar essa base para a construção de indicadores cienciométricos confiáveis e operacionalmente viáveis. Uma das possibilidades de amenização dessas limitações - que também deve ser retrospectiva - poderia ser através da ampliação do uso de formulários de preenchimento fechados, onde, por exemplo, o usuário tenha que selecionar uma revista de uma lista ao invés de digitá-la (na padronização de títulos, talvez o uso da listagem Qualis seja uma boa opção). Outro exemplo de sucesso em padronização de elementos bibliográficos que poderia ser avaliado, e quem sabe ter parte adaptada ao Currículo Lattes, é o MORE: Mecanismo On-line para Referências, uma ferramenta desenvolvida por Alves et al (2006), que produz automaticamente citações no texto e referências no formato $\mathrm{ABNT}^{6}$.

Com base no que foi denominado núcleo de periódicos da área de plasma, foi possível a identificação dos periódicos que estão disponibilizados através do Portal de Periódicos da CAPES (independentemente do acesso ser livre ou pago). Esse levantamento possibilitou visualizar as condições de disponibilidade e acessibilidade tanto do periódico quanto dos artigos publicados pelos pesquisadores. Nota-se que a maioria dos 62 periódicos encontra-se disponibilizada via Portal de Periódicos. No entanto, uma parcela menor, mas considerável, não se encontra disponibilizada no Portal. Dentre os periódicos não disponíveis, o que mais chama a atenção é o Materials Science Fórum, por ser o número 2 no ranking de periódicos onde os pesquisadores da área mais publicaram. Portanto, julga-se relevante que a área responsável pela política de aquisição da CAPES analise esse núcleo e verifique a necessidade de acrescentar ou excluir alguns dos periódicos listados. $O$

\footnotetext{
${ }^{6}$ Disponível em <http://robot.rexlab.ufsc.br/referencia/index.jsp>. Acesso em: 25 de fev. 2006.
} 
método utilizado para a definição de um núcleo de periódicos demonstrouse bastante promissor e funcional, e merece ser mais bem explorado e testado em outros estudos e áreas do conhecimento.

A determinação da qualidade dos artigos científicos publicados pelos pesquisadores na área de plasma teve como base a qualidade dos periódicos utilizados na veiculação desses artigos. Foi utilizada a Base de Dados Qualis: Classificação de Periódicos, Anais, Jornais e Revistas, versão 1.0 de 2004. Essa base foi concebida pela CAPES objetivando a avaliação dos programas de pós-graduação e, no entanto, pôde ser perfeitamente adotada e adaptada à presente pesquisa. O Qualis pode ser uma boa alternativa para amenizar alguns problemas e limitações encontradas em algumas bases de dados que tem sido utilizada para avaliação da produção científica (como é o caso das bases do Institute for Scientific Information - ISI). Pode ser uma alternativa viável, sobretudo, quando aplicada em países periféricos e áreas em que a ciência não é tão internacionalizada, como é o caso das áreas de ciências humanas, de algumas áreas da saúde, alguns ramos das ciências agrárias, dentre outras.

O presente trabalho seguiu o pressuposto de que "o periódico científico é, indiscutivelmente, o meio mais utilizado para a publicação dos resultados de pesquisa em todas as áreas do conhecimento" (BIOJONE, 2001 , p. 16). Desse modo, para este estudo julgou-se mais conveniente medir a produtividade com base na publicação de artigos científicos, já que se buscou entender melhor o comportamento desses (artigos e periódicos) após a implementação do Portal de Periódicos da CAPES. No entanto, é importante salientar que existem outros métodos para avaliar a produção científica, como por exemplo, através de livros publicados, capítulos de livros, apresentação de trabalhos em eventos, orientações, as teses e dissertações, depósito de patentes, etc. As análises desses indicadores também são de extrema importância para entender a dinâmica da produção e atividade científica, sendo importantes para trabalhos posteriores e abordagens complementares.

Analisando os dados de publicação ao longo do período de $1995 / 2005$, nota-se que praticamente todos os estados brasileiros tiveram aumento da produtividade, ou seja, aumentaram substancialmente o número de artigos publicados no período. Comparando os dois períodos (1995/1999 versus 2001/2005) nota-se que houve no último período um aumento de aproximadamente $54 \%$ nos artigos publicados. Trata-se de um aumento de produtividade muito expressivo a partir do ano 2000 (ano de implantação do Portal de Periódicos da CAPES).

Comparando a qualidade dos periódicos em que os pesquisadores da área de plasma mais publicaram nos períodos de 1995/1999 versus 2001/2005, nota-se um considerável aumento de artigos no segundo período (após implementação do Portal de Periódicos da CAPES). Os artigos publicados em periódicos com classificação $A$ no período de 2001/2005 cresceram aproximadamente $34 \%$ com relação ao período de 1995/1999. Os artigos publicados em periódicos com classificação $B$ cresceram aproximadamente $48 \%$ e os publicados em periódicos com 
classificação C cresceram aproximadamente 49\%. O crescimento dos artigos publicados com a classificação NC permaneceu praticamente estável (4\%) em comparação com o período anterior.

Com base neste estudo pode-se concluir que a área de plasma no Brasil encontra-se fortemente estruturada e amadurecida, com um potencial considerável para o desenvolvimento de inovações tecnológicas.

Nota-se, após a implementação do Portal de Periódicos da CAPES, indicadores expressivos de produtividade e qualidade dos artigos publicados pelos pesquisadores brasileiros na área de plasma.

Não há como saber o nível de influência do Portal de Periódicos da CAPES nos indicadores de produtividade e qualidade dos trabalhos publicados, já que são muitos os fatores que podem influenciar o sistema de pesquisa na área de plasma no Brasil. Os relevantes índices apresentados certamente são frutos de uma série de políticas públicas voltadas ao desenvolvimento da C\&T nacional, e, dentre elas, merecem destaque as políticas de informação implementadas pela CAPES, como é o caso do Portal de Periódicos da CAPES.

Certamente esses indicadores seriam diferentes se não houvesse sido implementado esse importante instrumento de apoio à pesquisa, sobretudo para estados e regiões que não dispõem de muitos recursos para investimento em C\&T. Assim, além da importância do Portal da CAPES no contexto nacional como um todo, o seu papel é também comprovado como um instrumento fundamental de democratização da informação em um país de desigualdades regionais gritantes.

\section{Referências}

ALVES, J. B. M. et al. Mecanismo on-line para referências versão 1.0 . Florianópolis: UFSC, 2006.

BIOJONE, M. R. Forma e função dos periódicos científicos na comunicação da ciência. 2001. 100f. Dissertação (Mestrado) - Escola de Comunicações e Artes - Departamento de Biblioteconomia e Documentação - USP, São Paulo, 2001.

BRADFORD, S. C. Sources of information on specific subjects. Engineering, V. 137, p. $85-86,1934$.

CALLON, M.; COURTIAL, J. P.; PENAN, H. Cienciometria: el estudio cuantitativo de la actividad científica: de la bibliometría a la vigilancia tecnológica. España: TREA, 1995.

CAPES - COORDENAÇÃO DE APERFEIÇOAMENTO DE PESSOAL DE NÍVEL SUPERIOR. Qualis: Classificação de periódicos, anais, jornais e revistas: versão 1.0 de 2004. Brasília, 2004. Disponível em <http://qualis.capes.gov.br/>. Acesso em: 10 maio. 2006.

CNPQ - CONSELHO NACIONAL DE DESENVOLVIMENTO CIENTÍFICO E TECNOLÓGICO. Plataforma Lattes: base de dados do diretório dos grupos de pesquisa no Brasil. Brasilia: CNPq, 1992. Disponível em <http://dgp.cnpq.br/buscaoperacional/>. Acesso em: 25 de fev. 2006. 
CNPQ - CONSELHO NACIONAL DE DESENVOLVIMENTO CIENTÍFICO E TECNOLÓGICO. Plataforma Lattes: currículo Lattes. Brasília, [199?]. Disponível em <http://buscatextual.cnpq.br/buscatextual/index.jsp> Acesso em 28 de fev. 2006.

RUSHTON, J. P.; MELTZER, S. 1981 apud MEADOWS, A. J. A comunicação científica. Brasília: Briquet de Lemos, 1999. (A referência completa do artigo citado por MEADOWS é RUSHTON, J. P.; MELTZER, S. Reasearch productivity, university revenue, and scholary impact (citations) of 169 British, Canadian and United States universities (1977). Scientometrics, n.3, p. $275-303,1981$.

FAPESP - FUNDAÇÃO DE AMPARO À PESQUISA DO ESTADO DE SÃO PAULO. Indicadores de ciência, tecnologia e inovação. São Paulo: FAPESP, 2001.

FERREIRA, S. P. et al. Recursos humanos disponíveis em ciência e tecnologia. In: LANDI, F. R. (Coord.). Indicadores de ciência, tecnologia e inovação em São Paulo 2004. São Paulo: FAPESP, 2005. cap. 4.

GREGOLIN, J. A. R. et al. Análise da produção científica a partir de indicadores bibliométricos. In: LANDI, F. R. (Coord.). Indicadores de ciência, tecnologia e inovação em São Paulo, 2004. São Paulo: FAPESP, 2005. cap. 5.

GUEDES, V.; BORSCHIVER, S. Bibliometria: uma ferramenta estatística para a gestão da informação e do conhecimento, em sistemas de informação, de comunicação e de avaliação científica e tecnológica. In: ENCONTRO NACIONAL DE CIÊNCIA DA INFORMAÇÃO, 6, 2005, Salvador. Proceedings... Salvador: UFBA, 2005.

MEADOWS, A. J. A comunicação científica. Brasília: Briquet de Lemos, 1999.

NELSON, R. (Ed). National innovation system: a comparative analysis. Nova York: Oxford University Press, 1993.

SPINAK, E. Indicadores cienciométricos. Ciência da Informação, Brasília, v. 27 , n. 2, p. 141-148, maio/ago. 1998.

VELHO, L. Indicadores de C\&T no Brasil: antecedentes e estratégia. Brasília, $1997^{7}$.

VOLPATO, G. L.; FREITAS, E. G. Desafios na publicação científica. Pesquisa Odontológica Brasileira, v. 17, p. 49-56, maio. 2003. 